\title{
Drug Development Process
}

National Cancer Institute

\section{Source}

National Cancer Institute. Drug Development Process. NCI Thesaurus. Code C142519.

The process by which an investig ational product is brought to the market through a series of preclinical and clinical testing, regulatory review, and approval. 